xviii

The part within the bracket may be written

$$
\begin{aligned}
& \{1 \quad+(n-1) \quad\} p_{11} p_{22} \ldots p_{n-1, n-1} \\
& +\{(n-1)+(n-1)(n-2)\} p_{11} \ldots p_{n-2, n-2} q_{n-1, n-1} \\
& +\left\{\left(\begin{array}{c}
n-1 \\
2
\end{array}\right)+(n-1)\left(\begin{array}{c}
n-2 \\
2
\end{array}\right)\right\} p_{11} \ldots p_{n-3, n-3} q_{n-2, n-2} q_{n-1, n-2} \\
& +\{(n-1)+(n-1)\} p_{11} q_{22} \ldots q_{n-1,2} \\
& +\{1+0\} q_{11} \ldots q_{n-1,1} \text {. }
\end{aligned}
$$

Adding vertically we get two expressions, exactly similar to the first term, the second expression being multiplied by $(n-1) p_{11}$. Hence the part within the brackets is equal to $1+(n-1) p_{11}$, and so the coefficient of $\alpha^{2} / 2$ ! in the moment G.F. is

$$
n p_{00}\left[\mathrm{l}+(n-\mathbf{1}) p_{11}\right] \text {. }
$$

Transferring to the mean as origin by subtracting $\left(n p_{00}\right)^{2}$, we derive the second moment $\mu_{2}$ about the mean, or squared standard deviation $\sigma^{2}$, as

$$
\sigma^{2}=n p_{00} q_{00}-n(n-1) p_{00}\left(p_{00}-p_{11}\right) .
$$

Thus the mean and standard deviation of this particular distribution are given in simple terms.

Example. For the distribution of one suit in a bridge hand, we have

$$
\begin{aligned}
& p_{00}=\frac{1}{4}, q_{00}=\frac{3}{4}, n=13, \quad p_{11}=\frac{12}{51}=\frac{4}{17} . \\
& \text { Hence } \quad \mu_{2}=\sigma^{2}=\frac{39}{16}-\frac{39}{68} \\
& =\frac{507}{272}=1 \cdot 86 \text {. } \\
& \text { and so } \quad \sigma=1 \cdot 36 \text {. }
\end{aligned}
$$

\title{
A Problem in Combinations
}

By A. C. Aitken.

1. If there are $n$ individuals $A_{1}, A_{2}, \ldots, A_{n}$, in how many ways can they be put into groups? For example, if there are three individuals $A, B, C$, they may be grouped as

$A+B+C ; A+(B+C), B+(C+A), C+(A+B) ; \quad(A+B+C)$, 
that is, in 5 ways, the respective subgroups, $1,3,1$ in number, corresponding to the partitions $1+1+1,1+2,3$ of the integer 3 . Hence $P(3)$, say, is 5 .

We shall obtain various expressions for $P(n)$, and shall place the problem in relation to other questions of analysis.

2. Following MacMahon we shall denote partitions, e.g. those of 3 above, by $1^{3}, 1^{1} 2^{1}, 3^{1}$; in general, if the integer $n$ is made up of a integers $a$, plus $\beta$ integers $b$, and so on, $a<b<\ldots \ldots$, we shall write the corresponding partition as

$$
a^{\alpha} b^{\beta} \ldots
$$

In the example given above, the partition $1^{1} 2^{1}$. leads to three subgroups. The 3 here is $3 ! /(1 ! 2 !)$, and in general it is easy to see, by the elementary theory of combinations, that the number of subgroups corresponding to the partition $a^{\alpha} b^{\beta} \ldots$ of $n$ is

$$
n ! /(a ! b ! \ldots a ! \beta ! \ldots) \text {. }
$$

Hence one answer to our problem is

$$
P(n)=\Sigma n ! /(a ! b ! \ldots \alpha ! \beta ! \ldots),
$$

the summation being over all partitions of the integer $n$; but this is not a very helpful expression.

3. We seek therefore a generating function, in which $P(n)$ shall appear as coefficient of $x^{n}$, or perhaps of $x^{n} / n !$.

Consider first groups made up of single units. If there are $r$ units in the partition (1) of $n$, then by (2) a factor $r$ ! will be required in the denominator. Hence unit groups will be represented by a generating function

this is, by $e^{x}$.

$$
\sum_{0}^{\infty} x^{r} / r !
$$

Next, groups of two. By (2), any 2 requires a 2 ! in the denominator for each time it occurs, and if it occurs $r$ times it requires an $r$ ! as well. The generating function for groups of two is therefore

$$
e^{x^{2} / 2 !}
$$


In the same way the generating function for groups of $r$ is

$$
e^{x^{r} / r !}
$$

Combining in multiplication all such generating functions, since groups of any size may be associated with groups of any other size, we have the required generating function for $P(n)$, namely

$$
e^{x+x^{2} / 2 !+x^{3} / 3 !+\cdots}=e^{e^{x}-1},
$$

and we derive the interesting fact that $P(n)$ is the coefficient of $x^{n} / n !$ in this expansion, that is, by Maclaurin's theorem,

$$
P(n)=\left[D^{n} e^{e^{x}-1}\right]_{x=0}, \text { where } D=\frac{d}{d x} .
$$

4. This result is connected with the procedure of repeatedly differentiating a function of a function. For example we have

$$
\begin{aligned}
& \frac{d}{d x} f(u)=f^{\prime}(u) \frac{d u}{d x}, \\
& \left(\frac{d}{d x}\right)^{2} f(u)=f^{\prime \prime}(u)\left(\frac{d u}{d x}\right)^{2}+f^{\prime}(u) \frac{d^{2} u}{d x^{2}}, \\
& \left(\frac{d}{d x}\right)^{3} f(u)=f^{\prime \prime \prime}(u)\left(\frac{d u}{d x}\right)^{3}+3 f^{\prime \prime}(u) \frac{d^{2} u}{d x^{2}} \cdot \frac{d u}{d x}+f^{\prime}(u) \frac{d^{3} u}{d x^{3}},
\end{aligned}
$$

and so on, and we notice that the coefficients $1,3,1$ in the third of these relations are the same as the numbers of subgroups in our first example.

In the expression for $\left(\frac{d}{d x}\right)^{n} f(u)$, let us put $f(u)=e^{u}, u=e^{x}$. Then the left hand side of the general relation of type (6) becomes

$$
\left(\frac{d}{d x}\right)^{n} e^{e^{x}}
$$

while the right hand side is a sum of terms involving

$$
e^{n x} e^{e^{x}}, e^{(n-1) x} e^{e^{x}}, \ldots, e^{x} e^{e^{x}}
$$

Putting $x=0$, we see that the sum of the numerical coefficients in the expansion of $\left(\frac{d}{d x}\right)^{n} f(u)$ is

$$
\left[e^{-1}\left(\frac{d}{d x}\right)^{n} e^{e^{x}}\right]_{x=0}=P(n) .
$$


5. Another set of relations, involving the operator $x \frac{d}{d x}$ or $x D$ which occurs in the theory of homogeneous differential equations, has the same coefficients as the set (6). For example we have

$$
\begin{aligned}
& (x D)^{2}=x^{2} D^{2}+x D, \\
& (x D)^{3}=x^{3} D^{3}+3 x^{2} D^{2}+x D, \text { etc. }
\end{aligned}
$$

Indeed, if $u=e^{x}$, the comparison between relations (6) and (8) becomes exact. For example the expression for $D^{4} f\left(e^{x}\right)$ is derived by term by term differentiation from that for $D^{3} f\left(e^{x}\right)$ by exactly the same formal operations as the expression for $(x D)^{4}$ is derived from that for $(x D)^{3}$; and so in general.

Inserting the operand $e^{x}$ in the expression for $(x D)^{n}$ corresponding to (8), and then putting $x=1$, we derive a new expression for $P(n)$,

$$
P(n)=\left[(x D)^{n} e^{x-1}\right]_{x=1},
$$

comparison of which with (5) yields the rather peculiar identity

$$
\left[D^{n} e^{e^{x}-1}\right]_{x=0}=\left[(\overline{x+1} D)^{n} e^{x}\right]_{x=0}
$$

6. Since

$$
e^{e^{x}-1}=e^{-1}\left(1+e^{x}+e^{2 x} / 2 !+e^{3 x} / 3 !+\ldots\right),
$$

and $P(n)$ is the coefficient of $x^{n} / n !$ in the expansion of this, we derive yet another expression,

$$
P(n)=e^{-1} \sum_{s=0}^{\infty}\left(s^{n} / s !\right)
$$

7. Next, let us write $s^{n}$ in terms of factorials $s, s(s-1)$, and so on. To do this, let a table of differences be formed from $0^{n}, 1^{n}, \ldots, n^{n}$, the differences of $0^{n}$ being denoted by $\Delta^{r} 0^{n}$. By the Gregory-Newton interpolation formula we have

$$
s^{n}=0^{n}+s \Delta 0^{n}+\frac{s(s-1)}{2 !} \Delta^{2} 0^{n}+\ldots+\left(\begin{array}{c}
s \\
n
\end{array}\right) \Delta^{n} 0^{n} .
$$

Substituting this in (11) for $s=0,1,2, \ldots$, we obtain

$$
\left.P(n)=e^{-1} \sum_{s=0}^{\infty}\left[\sum_{r=0}^{n} \Delta^{r} 0^{n} / \overline{(s-r !} ! r !\right)\right]
$$


xxii

On summation of expressions like $1 /(s-r)$ ! we obtain $e$ in each case, and so (12) gives

$$
\begin{aligned}
P(n) & =e^{-1} \sum_{r=1}^{n} e \Delta^{r} 0^{n} / r ! \\
& =\sum_{r=1}^{n} \Delta^{r} 0^{n} / r !
\end{aligned}
$$

which exhibits $P(n)$ as the sum of the "divided differences" of $0^{n}$. As an equivalent for (11) this was given by Herschel.

7. The numbers of subgroups also crop up in these divided differences of zero. For example, if $n=3$, the table of divided differences is

$\begin{array}{rrrr}0 & & & \\ 1 & 1 & & \\ 8 & 7 & & 1 \\ 27 & 19 & & \end{array}$

the 1, 3, 1 for this case appearing again. The theorem indicated here is a general one. To prove it we may operate on $x^{n}$ with $(x D)^{n}$ and its equivalent in (8), the result being an interpolation formula for $n^{n}$ in terms of $0^{n}$ and the differences of $0^{n}$.

8. One of the easiest ways of finding the first dozen or so numerical values of $P(n)$ is by means of the recurrence relation which $P(n)$ satisfies. This relation is

$$
\begin{aligned}
P(n+1) & =P(n)+n P(n-1)+\left(\begin{array}{l}
n \\
2
\end{array}\right) P(n-2)+\ldots+n P(1)+P(0) \\
& =(P+1)^{n}
\end{aligned}
$$

symbolically if, after expansion, exponents of $P$ are written as arguments. To prove this, we write

$$
P_{n+1}=e^{-1} D^{n+1}\left(e^{e^{x}}\right)=e^{-1} D^{n}\left[e^{x} \cdot e^{e^{x}}\right], \quad x=0 .
$$

Expanding the derivative of the product by Leibniz's theorem and then putting $x=0$, we have the result (14) at once.

Now the right side of (14) is in shape simply a GregoryNewton interpolation formula. Hence, since $P(0)=P(1)=1$, we see that if we construct a difference table from $P(1), P(2), P(3), \ldots$, then the values of $P(1), \Delta P(1), \Delta^{2} P(1), \ldots$ thereby given are simply $P(0), P(1), P(2), \ldots$, and so on. This gives perhaps the easiest way of all for finding the first several values of $P(n)$, namely 
to build up the table, entering each $P(r)$, when found, as a fresh difference $\Delta^{r} P(1)$ with which to begin a new line of differences. For example we have, for the first few values,

$\begin{array}{rrrrrr}P & \Delta & \Delta^{2} & \Delta^{3} & \Delta^{4} & \Delta^{5} \\ 1 & 1 & & & & \\ 2 & 3 & 2 & & & \\ 5 & 10 & 7 & 5 & 15 & \\ 15 & & 27 & & 67 & \\ 52 & 37 & 114 & & & \\ 203 & 151 & & & & \end{array}$

which puts in evidence the property mentioned.

9. The first ten values of $P(n)$ are

$\begin{array}{ccccrrrrrrc}n & 1 & 2 & 3 & 4 & 5 & 6 & 7 & 8 & 9 & 10 \\ P(n) & 1 & 2 & 5 & 15 & 52 & 203 & 877 & 4140 & 21147 & 115975 .\end{array}$

Inspection shows that if $n$ is a prime number $>1, p$ say, then $P(p)-2$ is divisible by $p$. For example $877-2$ is divisible by 7 .

This is a result easily proved. For $\Delta 0^{p}=1$, and $\Delta^{p} 0^{p}=p$ !, so that the $p^{\text {th }}$ divided difference of $0^{p}$ is also 1 . As for the differences of $0^{p}$ of order $r$, where $1<r<p$, it is an instant deduction from Fermat's theorem that

$$
\begin{array}{rlr}
\Delta^{r} 0^{p} & \equiv \Delta^{r} 0^{1} & \bmod p, \\
& =0, & 1<r<p .
\end{array}
$$

To obtain the divided differences, which must be integers, we divide the ordinary differences $\Delta^{r} 0^{p}$ by $r$ !, which does not contain $p$, since $p$ is a prime greater than $r$. Hence the divided differences for $1<r<p$ are also divisible by $p$. On summing these divided differences in (13), we obtain for $P(p)$ a multiple of $p$, plus 1 from each end term. Hence, as stated, $P(p)-2$ is divisible by $p$.

10. After these various diversions, it would have been pleasing to find an asymptotic expression to represent $P(n)$ for large values of $n$, but this has not so far materialized. The function $n^{\frac{1}{2} n}$ gives a fair representation for small values, up to $n=8$; for example $P(8)=4140$, while $8^{4}=4096$. For higher values $P(n)$ increases more rapidly; for example $P(10)=115975$, while $10^{5}=.100000$. 